$4^{\circ}$ died. GZ 103 (5 Hormone and Metabolie Resegareh

Editors-in-Chief

R. Levine Duarte

E. F.Pfeiffer Ulm

Assistant-Editors

R. J. Mahler Duarte

R. Ziegler Ulm

VOL. 5

1973

258 Figures in

295 Single figures

208 Tables

Georg Thieme Verlag • Stuttgart 


\section{TABLE OF CONTENTS}

Kumar, D. and L.V. Miller

The Prevalence of Proinsulin-Specific Antibodies in Diabetic Patients

Berger, W., H. Göschke, J. Moppert and H. Künzli

Insulin Concentrations in Portal Venous and Peri. pheral Venous Blood in Man Following Administration of Glucose, Galactose, Xylitol and Tolbutamide

Beyer, J., E. Haupt, U. Cordes, J. Kutschera and $K$. Schöffling

Different Amounts of Insulin Secretion Following an Equal Total Decrease of Blood Glucose as an Indication of Possible Extrapancreatic Activities of Different Sulfonylurea Drugs

Hebold, G. and H. Bleuel

Various Blood Glucose Values in the Rat

Sutter-Dub, M.-Th., R. Leclercq, J.M. Felix, $R$. Jacquot and B.Ch.J. Sutter

Serum Progesterone and Immuno-Reactive Insulin Levels in the Pregnant Rat

Frohman, L.A., E.E. Muller and D. Cocchi Central Nervous System Mediated Inhibition of Insulin Secretion Due to 2-Deoxyglucose ....

Rossini, A.A. and M.G. Buse

The Effect of L-Dopa on Insulin Release by Isolated Pancreatic Islets of the Rat

Schatz, H., V. Maier, M. Hinz, M. Schleyer, C. Nierle and E.F. Pfeiffer

Hypophysis and Function of Pancreatic Islets

Akerblom, H.K., J.M. Martin and G.L. Garay

Relative Role of Cortisone and Growth Hormone on Glucose Intolerance and Insulin Secretion in Rat

Batchelor, B.R. and J.S. Stern

The Effect of Growth Hormone upon Glucose Metabolism and Cellularity in Rat Adipose

Tissue

Imura, H., Y. Nakai, S. Matsukura and H. Matsuyama

Effect of Intravenous Infusion of L-Dopa on Plasma Growth Hormone Levels in Man

Pogátsa, G., A. Káldor and K. Nekám

Effect of Leucine on Carbohydrate Metabolism of the Liver

Hinman, D.J. and D.R. Griffith

Effects of Ventromedial Hypothalamic Lesions on Thyroid Secretion Rate in Rats

Wang, Jiin-bor, J.C. Rogler and D.P. Doolittle Effect of Alloxan on Calcium Metabolism in Mice and Rats
Naithani, V.K.

Immunoassays of the Nine Synthetic Porcine

1 C-Peptide Sequences

Maturo III, J.M. and S. Diloff

Interaction between Amino Acids and Insulin

Aynsley-Green, A. and K.G.M.M. Alberti

The Importance of the Guanidine Group in the Insulin-Stimulatory Effect of Amiloride Hydrochloride

Renner, R., K.D. Hepp, R.E. Humbel and E.R. Froesch

Mechanism of the Antilipolytic Action of

NSILA-S: Inhibition of Adenylate Cyclase Activity in Lipocyte Ghosts

Serrano-Rios, M., J. Mato, M. Oya, L. Larrodera, $F$. Hawkins and F. Escobar

Failure to Find Ig-G Insulin Antibodies in

Down's Syndrome

Virkkunen, P., J. Leppäluoto, J. Ranta and

H. Lybeck

Preparation of ${ }^{125}$ I-Labelled Thyrotrophin-

Releasing Hormone

Verdy, M. and J. Marc-Aurele

Fasting in Obese Females: Plasma Renin after Glucose Refeeding

Guy-Grand, B. and R. Assan

Lipolytic Activity of Synthetic (1-23) Glucagon in Vitro

Das, $I$.

$\mathrm{Mg}^{2+}$-Stimulated ATPase Activity in Starved and Diabetic Rat Heart

Toccafondi, R.S. and S.B. Sufi

Thyroid Hormone Binding to the Anterior Pituitary Gland

Radó, J.P.

Falsely High Fluorescence in Cortisol Determina-

tion Due to the Carbamazepine

Stolecke, $H$.

Normal Values of Free Urinary Cortisol in Infants and Children

41 Keller, P.J. and C. Gerber

Effect of Sublingual, Intravenous and Intramuscular Administration of Synthetic LHReleasing Hormone in the Human

Berk, J., J. Hagen, R. Koo, S. Nomoto and M. Rupright

Pulmonary Arteriovenous Shunting Due to Epinephrine

A. Cohen

Plasma Corticosterone Concentration in the

Foetal Rat 
Lev-Ran, A. and L. Ratt

Insulin Binding and Free Insulin in Serum of Insulin-Treated Diabetics

Fernandez-Cruz (Jr.), A., O. Garcia Hermida, M. Lugue Otero and R.E. Catalán

Human Bile Insulin Sephadex Fractions ....

Franckson, J.R.M. and H.A. Ooms

The Glomerular Clearance of Exogenous Insulin

Minaire, Y., A. Pernod, J.C. Vincent-Falquet and M. Mottaz

Comparison of Carbohydrate Utilization in

Running and Shivering Dogs

Hiatt. N., L. Morgenstern, M.B. Davidson, G. Bonorris and A. Miller

Role of Insulin in the Transfer of Infused

Potassium to Tissue

Felix, J.M., B.Ch.J. Sutter and R. Jacquot Inhibitors of Proteolysis and Glucose Uptake by Diaphragm Muscle in Vitro under Insulin Action

Heißmeyer, H., U. Stein, R. Grub, W. Reutter and $R$. Lesch

The Effect of Galactosamine on the Activity of Lysosomal Peptidehydrolases of the Liver Depending on the Age of Rats

Domschke, S. and H.D. Söling

Polyamine Metabolism in Rat Liver

Bolt, H.M. and H. Remmer

The Fate of Mestranol (17a-Ethynylestradiol-2-Methyl Ether) in the Isolated Perfused Rat Liver

Gacs, G., E. Kun and Zs. Czirbesz

Growth Hormone Secretion in Children during Corticosteroid Treatment. Responses to Arginine

Szabo, S., K. Kovacs, B.D. Garg, B. Tuchweber and $G$. Lazar

Effect of Hypophysectomy and Cyproterone Acetate on the Liver Ultrastructure as well as on Hexobarbital Anesthesia and Zoxazolamine Paralysis in Rats

J.J. Buneaux and J.C. Gounelle

Influence of Calcium and Somatotropin on D-Xylose Uptake by the Isolated Diaphragm of Hypophysectomized Rats

Le Roith, D. and B. Pimstone

The Use of Simultaneously Administered ${ }^{47} \mathrm{Ca}$ and ${ }^{45} \mathrm{Ca}$ in the Study of Calcium Kinetics in the Rat

\section{J.P. Barlet}

The Influence of Gastrin on the Fecal and Urinary Excretion of Water, Calcium, Magnesium, and Inorganic Phosphorus in Sheep .... 124
Castillo, C.E. and F. Beas

Crop Sac Stimulating Activity and Mammary Inhibition Produced by a Human Placental Protein

Garmendia, F., E. Kesserü and L.A. Llerena

Serum LH Concentration in Women under

Contraceptive Treatment with Estrogen-Free Progestagens

Hetenyi Jr., G. and R.L. Singhal

Effect of Insulin on Cerebral Adenyl Cyclase and Phosphodiesterase

Krause, U., H. Puchinger and A. Wacker

Comparison of Various Filter Materials for Insulin Immunoassay

Berne, C., S.E. Brolin and A. Agren

Influence of Ischemia on the Levels of Reduced Pyridine Nucleotides in the Pancreatic Islets

Graf, L., G. Hajos, A. Patthy and G. Cseh

The Influence of Deamidation on the Biological Activity of Porcine Adrenocorticotropic Hormone (ACTH)

Iturriza, F.C., H. Levitin and F. Estivariz A New Extrapigmentary Effect of AlphaMSH. Promotion of Sodium Uptake in the Heart of Toads and Rats

Gozariu, L. and O. Florescu

Calcitonin and Glucose Uptake by Isolated

Rat Diaphragm

Christiansen, Aa.H.

Radioimmunoelectrophoresis in the Determination of Insulin Binding to IgG.

Methodological Studies

Barling, $P$. and Anne Beloff-Chain

Studies on the Administration of Glucagon and Insulin Antibodies to Rats

Ahuja, M.M.S., S.P. Gupta and V.K. Sehgel Efficacy of Glibenclamide in Maturity-Onset Diabetics as Maintenance Therapy ..........

Stubbe, $P$., H. Mentzel and $H$. Wolf

Growth Hormone Fluctuations in the Paramortal Period

Kostyo, J.L. and D.F. Nutting

Acute In Vivo Effects of Growth Hormone on Protein Synthesis in Various Tissues of Hypophysectomized Rats and their Relationship to the Levels of Thymidine Factor and Insulin in the Plasma

Rabkin, R., B.L. Pimstone and L. Eales Autoradiographic Demonstration of Glomerular Filtration and Proximal Tubular Ab. sorption of Growth Hormone ${ }^{125} \mathrm{I}$ in the Mouse 
Delespesse, G., J. Duchateau, B. Kennes, A. Govaerts and P.A. Bastenie

Thyroxine Elimination by Peritoneal Dialysis in Experimental Thyrotoxicosis

Kissebah, A.H., P. Harrigan and V. Wynn Mechanism of Hypertriglyceridaemia Associated with Contraceptive Steroids

Guisard, D., A. Bach, G. Debry and P. Métais Comparison of the Metabolic Effects of Short and Medium Even-Chain Fatty Acids $\left(\mathrm{C}_{9}\right)$ Triglycerides in Dogs

Frandsen, E.K. and A.J. Moody

Lipolytic Action of A Newly Isolated Vasoactive Intestinal Polypeptide

Whitfield, J.F., J.P. MacManus and D.J. Gillan The Calcium-Dependent Stimulation of Thymic Lymphoblast DNA Synthesis and Proliferation by a Low Concentration of Cortisol

Németh, S., A. Straková and M. Viges

The Role Played by Adrenal Hormones in the Increase of Liver Tyrosine Aminotransferase Activity of Rats Subjected to Trauma

Parvez, S., D. Gripois and H. Parvez Urinary Excretion of Adrenalin, Noradrenalin and Vanylmandelic Acid in Rats during Pregnancy and Post-Partum. Effects of Niamide and Reserpine Administration

Beatty, Clarissa H., R.M. Bocek and M.K. Young Effect of Oxytocin and Epinephrine on the Adenylate Cyclase Activity of Myometrium from Pregnant Rhesus Monkeys

Kilbert, L.H. Jr., A.R. Goldfarb and P.P. Foà A Micro-Method for the Measurement of Pharmacologically Induced Changes in Purine Derivatives

Matzkies, F., W. Grabner, H. Scharrer, H. Bickel and $G$. Berg

Insulin Secretion after Long-Term Infusion of Xylithol

Debry, G., R. Rohr, L. Mejean and D. Guisard The Effect of Fractionating the Caloric Intake on Weight, Serum Lipids, Post-Prandial Blood Sugar, Serum Insulin and the Oral Glucose Tolerance Test in the Normal Adult

Lemonnier, D., J.-P. Suquet, R. Aubert, G. Rosselin

Long Term Effect of Mouse Neonate Food Intake on Adult Body Composition, Insulin and Glucose Serum Levels

Muggeo, M., D. Fedele, A. Tiengo and G. Crepaldi

Growth Hormone Secretion in Primary Endogenous Hypertriglyceridemia
Nakagawa, K., K. Mashimo

Suppression of Exercise-Induced Growth Hormone Release with Dexamethasone

225

Smythe, G.A. and L. Lazarus

Blockade of Serotonin Induced GH Secretion in Rats by Melatonin and Cyproheptadine ...

Baumann, J.B., M. Stahl, P.W. Nars and

$J$. Girard

Effect of Collagenase on Human Growth Hormone and ACTH

Dubois, M.P. and L. Graf

Demonstration by Immunofluorescence of the Lipotropic Hormone/LPH in Bovine, Ovine and Porcine Adenohypophysis

Erle, G., G. Federspil, D. Casara, M. Zaccaria and $C$. Scandellari

Effects of L-Triiodothyronine on Insulin Secre-

tion in Man

Bieler, E.U., R.J. van Rooyen, E.J.P. de Bruin and J.M.C. Hoog

A Radioimmunoassay Technique for Human

204 Calcitonin (HC) in Plasma Avoiding the Nonspecific Interferences of Plasma Proteins on the Tracer Binding

Ardaillou, R., Francoise Paillard, J. Sraer and $G$. Vallée

Compared Kinetics of Salmon and Human

Radioiodinated Calcitonins in Man

Kinson, G.A. and Chung-ching Liu

Diurnal Variation in Plasma Testosterone of the Male Laboratory Rats

Bolt, H.M. and H. Kappus

Effect of Clotrimazol Treatment on the Demethylation of Mestranol

Hesch, R.D., S. Woodhead, M. Huefner and H. Wolf

Gastrointestinal Stimulation of Calcitonin in Adults and Newborns

Boden, G., V. Dinoso and O.E. Owen

Immunological Comparison of Natural and Synthetic Secretins

Weir, G.C., R.C. Turner and D.B. Martin Glucagon Radioimmunoassay Using Antiserum 30K: Interference by Plasma

Lefebvre, P., A. Luyckx and Z.M. Bacq

Effects of Denervation on the Metabolism and the Response to Glucagon of White Adipose Tissue of Rats

Dudl, R.J., R.L. Lerner, J.W. Ensinck and R.H. Williams

The Effect of Secretin on Pancreatic Glucoregulatory Hormones in Man 
Gilardeau, C. and M. Chrétien

Lipolytic Activity of Rat Pituitaries in Vitro

253

Müller, O.A., W. Pister, P. Schwandt, P.C.

Scriba and $P$. Weisweiler

Biological ACTH Activity of Lipid Mobilizing

Peptide Fractions from Hog Pituitaries ........ 258

Rajaniemi, H. and T. Vanha-Perttula

Evidence for LH and FSH Binding Protein(s) in

Human and Rat Serum

v.z. Mühlen, $A$. and $J$. Köbberling

Effect of Testosterone on the LH and FSH

Release Induced by LH-Releasing Factor (LRF)

in Normal Men

Kley, H.K., J. Herrmann, K.D. Morgner and

H.L. Krüskemper

Effects of Testosterone Oenanthate on Plasma

Concentrations of Thyroxine, Cortisol, Testos-

terone and Hormone Binding Proteins in $\mathrm{Pa}$ -

tients with Hypogonadism

Kissebah, A.H., P. Harrigan, P.W. Adams and V. Wynn

Effect of Methandienone on Free Fatty Acid and Triglyceride Turnover in Normal Females

Ács, Zsuzsanna and E. Stark

The Role of Transcortin in the Distribution

of Corticosterone in the Rat 279

Németh, S., M. Vigas and A. Straková

Time and Level of Increased Plasma Corticosterone Necessary for Induction of Liver Tyrosine Aminotransferase in Rats Subjected to Trauma

Hilgertová, Jiłina and J. Šonka

Effects of Cold Exposure, Thyroid Extract, Methylthiouracil and Dehydroepiandrosterone on Glucose 6-Phosphate and 6-Phosphogluconate Dehydrogenases in Rat Liver and Erythrocytes

Faglia, G., C. Ferrari, P. Beck-Peccoz, A. Spada, P. Travaglini and B. Ambrosi

Reduced Plasma Thyrotropin Response to Thyrotropin Releasing Hormone After Dexamethasone Administration in Normal Subjects

Werner, Sigbritt and H. Löw

Stimulation of Lipolysis and Calcium Accumulation by Parathyroid Hormone in Rat Adipose Tissue in Vitro after Adrenalectomy and Administration of High Doses of Cortisone Acetate

Black, H.E. and C.C. Capen

Plasma Calcitonin-Like Activity and Urinary Cyclic Adenosine Monophosphate during Pregnancy, Parturition, and Lactation in Cows with Parturient Hypocalcemia
Rastogi, G.K., R.J. Dash and M.K. Sinha Glucose, FFA, IRI and GH Response to I.V. Glibenclamide

Pointner, H., J.P. Accary, J. Vatier, M. Du-

brasquet and $S$. Bonfils

Detection of Gastrin in Normal Pancreas by

Radioimmunoassay

Curtis-Prior, P.B.

Lipolytic Activity of Serum and of Products of Serum Ultrafiltration

305

Koch, B., B. Bucher and C. Mialhe

Influence of Median Eminence Extracts on

Neosynthesis and Release of ACTH

Morgner, K.D. and H.L. Krüskemper

Suppression of the Hypothalamic-Pituitary-

Adrenal-Axis with Dexamethasone

307

Andresen, $\phi$.

Development of a Radioimmunoassay for

5a-Androst-16-ene-3-one

Radó, J.P.

Clinical Use of Additive Antidiuretic Action of Carbamazepine and Chlorpropamide ......

Chabot, V. and J.-P. Felber

Neutral Fat Infusion

Belz, G.G. and U.R. Kleeberg

Inhibition of $\mathrm{Na}^{+}-\mathrm{K}^{+}$-ATPase and ${ }^{86} \mathrm{Rb}$-up-

take by Canrenone

Metzger, P., P. Franken and E.O. Balasse

Permissive Role of Glucose on the Insulinotropic Effect of Ketone Bodies in Vivo ....

Dieterle, P., B. Birkner, K.-H. Gmeiner,

$P$. Wagner, F. Erhardt, J. Henner and

C. Dieterle

Release of Peripherally Stored Insulin During Acute Muscular Work in Man

Vigaš, M., S. Németh and Jana Jurčvixová The Mechanism of Trauma-Induced Inhibition of Insulin Release

Krause, U., H. Puchinger and A. Wacker Inhibition of Glucose-Induced Insulin Secretion in Trypsin-Treated Islets of Langerhans

Das, $I$.

Effect of Diabetes and Insulin on the Rat Heart Adenyl Cyclase, Cyclic AMP Phosphodiesterase and Cyclic AMP

Taketomi, S., M. Tsuda, T. Matsuo, H. Iwatsuka and $Z$. Suzuoki

Alterations of Hepatic Enzyme Activities in KK and Yellow KK Mice with Various Diabetic States 
August, G.P., R.F. Cheng, W. Hung and J.C. Houck

Fibroblast Proliferative Activity in the Sera of Growth Hormone Deficient Patients

W. Geiger

Radioimmunological Determination of Human Chorionic Gonadotropin, Human Placental Lactogen, Growth Hormone and Thyrotropin in the Serum of Mother and Child during the Early Puerperium

Fredholm, B.B. and B. Persson

Actions of Human Chorionic Somatomammotropin (HCS) in Dog Subcutaneous Adipose Tissue In Situ

Aranda, Ana, E. Blázquez and E. Herrera

Liver Components, Blood Glucose and Ketone

Bodies in Fed and Starved Suckling Rats

York, D.A. and G.A. Bray

Adipose Tissue Metabolism in Six Week Old

Fatty Rats

Hebold, G. and H. Bleuel

Sex-Linked Blood-Glucose Pattern in Rats Under Varying Test Conditions

Mims, R.B.

Plasma ACTH in the Adrenalectomized Rat

Lefauconnier, Jeanne-Marie, Christiane Portemer, G. de Billy and Fernande Chatagner Effect of Cortisol on the Level of Pyridoxal Phosphate-Dependent Enzymes in the Rat Liver: New Supports for Antagonism between Female Sex Hormones and Cortisol

Fontaine, Y.-A., C. Salmon, E. Fontaine-Bertrand and $N$. Delerue-Le Belle

Age-Related Changes in FSH- and in LH-Sensitive Ovarian Adenyl Cyclase from Prepubertal Rats

Fischer, J.A., U. Binswanger, A. Fanconi, $R$. Illig, K. Baerlocher and A. Prader

Serum Parathyroid Hormone Concentrations in Vitamin D Deficiency Rickets of Infancy: Effects of Intravenous Calcium and Vitamin D

von Berger, L., S. Raptis, G. Stüber, Th.

Schmidt and E.F. Pfeiffer

Radioimmunologically Measurable Gastrin

Level after "Sham Eating" in Man

Kidson, W. and L. Lazarus

The Glycaemic Stimulus to Early Phase Insulin Release: Quantitation of Beta Cell Function

Linde, $J$. and T. Teckert

Increase of Insulin Concentration in MaturityOnset Diabetics by Phentolamine (Regitine ${ }^{\circledR}$ ) Infusion
Jansen, F.K. and L. Herberg

Immune Reactions to Fractions of Crystallized Insulin. III. Relation Between Glucose Tolerance, Purification of Insulin and Insulin Antibodies

Kaess, H., W. Ehlers and A. Burkhardt

Distribution of Electrolytes and Insulin Content in the Pancreas of Potassium Deficient Rats

Gunnarsson, $R$. and C. Hellerström

Acute Effects of Alloxan on the Metabolism and Insulin Secretion of the Pancreatic B-Cell

404

Asplund, $K$.

Effects of Glucose on Insulin Biosynthesis in

Foetal and Newborn Rats

Tung, A.K.

Biosynthesis of Avian Glucagon: Evidence for a Possible High Molecular Weight Biosynthetic Intermediate

Santeusanio, F., G.R. Faloona and R.H. Unger Inhibition of Alanine-Stimulated Glucagon Secretion by Secretin in the Dog

Langslow, D.R.

The Action of Gut Glucagon-Like Immunoreactivity and Other Intestinal Hormones in Lipo-

lysis in Chicken Adipocytes

Curtis-Prior, P.B.

Studies on the Action of High Doses of Insulin and Anti-Insulin Serum (AIS) on Basal and

Serum-Induced Lipolysis in the Isolated Fat

Cell

Goebell, H. and P. Schmitz-Moormann

The Effect of Ethionine on the Energy Producing Metabolism in the Rat Pancreas. I. The Influence on the Activities of Key Enzymes of

Various Metabolic Pathways

Gasquet, $P$. de and E. Pequignot

Changes in Adipose Tissue and Heart Lipoprotein Lipase Activities and in Serum Glucose, Insulin and Corticosterone Concentrations in Rat Adapted to a Daily Meal

Bolzano, K., F. Sandhofer, S. Sailer and H. Braunsteiner

Studies on Cholesterol Turnover in Hypercholesterolemic Subjects

Haft, D.E.

Role of Plasma Triglyceride Turnover in Deposition of Newly Synthesized Fatty Acid in Adipose Tissue

Massary, F., F. Camanni and G.M. Molinatti

Amantadine Enhancement of L-DOPA Induced Growth Hormone Stimulation
Makris, Anastasia and S.N. Gershoff

Growth Hormone Levels in Vitamin $\mathrm{B}_{6}$-Deficient

Rats 
de Gennes, J.L., B. Bigorie, C. Bertrand and $J$. Truffert

Antidiuretic Effect of Clofibrate in Therapy of Diabetes Insipidus

Baráth, $P$. and G. Csaba

Effect of Pinealectomy on the Tritiated Serotonin Uptake of the Adrenal Medulla

Kramer, M.W., Shirley E. Pearson and S.M.

Michaelson

Plasma Disappearance of Radioiodinated Dog

Growth Hormone in the Dog

Ditzel, $J$.

Importance of Plasma Inorganic Phosphate on Tissue Oxygenation During Recovery from Diabetic Ketoacidosis
Carducci, A., Artenisio M. Sprovieri, M.C. Albanese, $V$. Allegro, $F$. Ragonese and $V$. Corsi

Effects of Propranolol on HGH Secretion after

461 Endovenous Tolbutamide Load in Obese Subjects

Zimmermann-Telschow, $H .$, W. Wiegelmann,

466 H.G. Solbach and H. Zimmermann

The Different Responses of Blood Amino Acids and Blood Glucose to the Administration of Insulin to Patients with Acromegaly

470 Barlet, J.P., D. Goeneche, H. Jourde and J. Michelot

Calcitonin and Iodinated Hormones in Sheep 


\section{AUTHOR'S INDEX}

A

Accary, J.P. 303

Acs, Zsuszanna 279

Adams, P.W. 275

Agren, A. 141

Ahuja, M.M.S. 160

Akerblom, H.K. 34

Albanese, M.C. 473

Alberti, K.G.M.M. 55

Allegro, V. 473

Ambrosi, B. 289

Andresen, $\emptyset .307$

Aranda, Ana 350

Ardaillou, R. 232

Asplund, K. 410

Assan, R. 60

Aubert, R. 223

August, G.P. 340

Aynsley-Green, A. 55

B

Bach, A. 191

Bacq, Z.M. 245

Baerlocher, K. 381

Balasse, E.O. 313

Baráth, P. 466

Barlet, J.P. 124, 475

Barling, P. 154

Bastenie, P.A. 176

Batchelor, B.R. 37

Baumann, J.B. 228

Beas, F. 129

Beatty, Clarissa H. 213

Beck-Peccoz, P. 289

Beloff-Chain, Anne 154

Belz, G.G. 312

Berg, G. 221

Berger, L. von 386

Berger, W. 4

Berk, J. 65

Berne, C. 141

Bertrand, C. 461

Beyer, J. 9

Bickel, H. 221

Bieler, E.U. 231

Bigorie, B. 461

Billy, G. de 372

Binswanger, U. 381

Birkner, B. 316

Black, H.E. 297

Blázquez, E. 350

Bleuel, H. 14, 361

Bocek, R.M. 213

Bcden, G. 237

Bolt, H.M. 101, 234

Bolzano, K. 444

Bonfils, S. 303

Bonorris, G. 84

Braunsteiner, H. 444

Bray, G.A. 355

Brolin, S.E. 141

Bruin, E.J.P. de 231

Burkhardt, A. 401

Bucher, B. 306

Buneaux, J.J. 113

Buse, M.G. 26

C

Camanni, F. 454
Capen, C.C. 297

Carducci, A. 473

Casara, D. 230

Castillo, C.E. 129

Catalán, R.E. 70

Chabot, V. 310

Chatagner, Fernande 372

Cheng, R.F. 340

Chrétien, M. 253

Christiansen, Aa. H. 147

Chung-Ching Liu 233

Cocchi, D. 21

Cohen, A. 66

Cordes, U. 9

Corsi, V. 473

Crepaldi, G. 224

Csaba, G. 466

Cseh, G. 142

Curtis-Prior, P.B. 305, 432

Czirbesz, Zs. 106

D

Das, I. 61, 330

Dash, R.J. 303

Davidson, M.B. 84

Debry, G. 191, 222

Deckert, T. 391

Delerue-LeBelle, N. 376

Delespesse, G. 176

Dieterle, C. 316

Dieterle, P. 316

Diloff, S. 54

Dinoso, V. 237

Ditzel, J. 471

Domschke, S. 97

Doolittle, D.P. 51

Dubois, M.P. 229

Dubrasquet, M. 303

Duchateau, J. 176

Dudl, R.J. 250

E

Eales, L. 172

Ehlers, W. 401

Ensinck, J.W. 250

Erhardt, F. 316

Erle, G. 230

Escobar, F. 57

Estivariz, F. 143

F

Faglia, G. 289

Faloona, G.R. 425

Fanconi, A. 381

Fedele, D. 224

Federspil, G. 230

Felber, J.-P. 310

Felix, J.M. 18, 89

Fernandez-Cruz (Jr.), A. 70

Ferrari, C. 289

Fischer, J.A. 381

Fiorescu, O. 145

Foà, P.P. 216

Fontaine, Y.-A. 376

Fontaine-Bertrand, E. 376

Franckson, J.R.M. 75

Frandsen, E.K. 196

Franken, P. 313

Fredholm, B.B. 347

Froesch, E.R. 56
Frohman, L.A. 21

G

Gacs, G. 106

Garay, G.L. 34

Garcia Hermida, O. 70

Garmendia, F. 134

Garg, B.D. 109

Gasquet, P. de 440

Geiger, W. 342

Gennes, J.L. de 461

Gerber, C. 64

Gershoff, S.N. 457

Gilardeau, C. 253

Gillan, D.J. 200

Girard, J. 228

Gmeiner, K.-H. 316

Godenèche, D. 475

Goebell, H. 435

Goldfarb, A.R. 216

Göschke, H. 4

Gounelle, J.C. 113

Govaerts, A. 176

Gozariu, L. 145

Grabner, W. 221

Gráf, L. 142, 229

Griffith, D.R. 48

Gripois, D. 207

Grub, R. 93

Guisard, D. 191, 222

Gunnarsson, R. 404

Gupta, S.P. 160

Guy-Grand, B. 60

$\mathbf{H}$

Haft, D.E. 449

Hagen, J. 65

Hajos, G. 142

Harrigan, P. 184, 275

Haupt, E. 9

Hawkins, F. 57

Hebold, G. 14, 361

Heißmeyer, H. 93

Hellerström, C. 404

Henner, J. 316

Hepp, K.D. 56

Herberg, L. 395

Herrera, E. 350

Herrmann, J. 180, 271

Hesch, R.D. 235

Hetenyi Jr., G. 139

Hiatt, N. 84

Hilgertová, Jifina 286

Hinman, D.J. 48

Hinz, M. 29

Hoog, J.M.C. 231

Houck, J.C. 340

Huefner, M. 235

Humbel, R.E. 56

Hung, W. 340

I

Illig, R. 381

Imura, H. 41

Iturriza, F.C. 143

Iwatsuka, H. 333

J

Jacquot, R. 18,89

Jansen, F.K. 395 
Jiin-Bor Wang 51

Jourde, H. 475

Juř̌oviZová, Jana 322

$\mathbf{K}$

Kaess, H. 401

Káldor, A. 45

Kappus, H. 234

Keller, P.J. 64

Kennes, B. 176

Kesserï, E. 134

Kidson, W. 387

Kilbert Jr., L.H. 216

Kinson, G.A. 233

Kissebah, A.H. 184, 275

Kleeberg, U.R. 312

Kley, H.K. 271

Köbberling, J. 266

Koch, B. 306

Koo, R. 65

Kostyo, J.L. 167

Kovacs, K. 109

Kramer, M.W. 470

Krause, U. 140, 325

Krüskemper, H.L. 180, 271, 307

Kumar, D. 1

Kun, E. 106

Künzli, H. 4

Kutschera, J. 9

L

Larrodera, L. 57

Langslow, D.R. 428

Lazar, G. 109

Lazarus, L. 227, 387

Leclercq, R. 18

Lefauconnier, Jeanne-Marie 372

Lefebvre, P. 245

Lemonnier, D. 223

Leppäluoto, J. 58

Lerner, R.L. 250

Lesch, R. 93

Lev-Ran, A. 67

Levitín, H. 143

Linde, J. 391

Lis, M. 253

Liu, Chung-Ching 233

Llerena, L.A. 134

Löw, H. 292

Luque Otero, M. 70

Luyckx, A. 245

Lybeck, H. 58

M

MacManus, J.P. 200

Maier, V. 29

Makris, Anastasia 457

Marc-Aurele, J. 59

Martin, D.B. 241

Martin, J.M. 34

Mashimo, K. 225

Massara, F. 454

Mato, J. 57

Matsukura, S. 41

Matsuo, T. 333

Matsuyama, H. 41

Maturo III., J.M. 54

Matzkies, F. 221

Mejean, L. 222

Mentzel, H. 163

Métais, P. 191

Metzger, P. 313
Mialhe, C. 306

Michaelson, S.M. 470

Michelot, J. 475

Miller, A. 84

Miller, L.V. 1

Mims 368

Minaire, Y. 80

Molinatti, G.M. 454

Moody, A.J. 196

Moppert, J. 4

Morgenstern, L. 84

Morgner, K.D. 271, 307

Mottaz, M. 80

Muggeo, M. 224

Muihlen, A. von zur 266

Muller, E.E. 21

Müller, O.A. 258

$\mathbf{N}$

Naithani, V.K. 53

Nakagawa, K. 225

Nakai, Y. 41

Nars, P.W. 228

Nékám, K. 45

Németh, S. 204, 283, 322

Nierle, C. 29

Nomoto, S. 65

Nutting, D.F. 167

0

Ooms, H.A. 75

Owen, O.E. 237

Oya, M. 57

$\mathbf{P}$

Paillard, Francoise 232

Parvez, H. 207

Parvez, S. 207

Patthy, A. 142

Pearson, Shirley, E. 470

Pequignot, E. 440

Pernod, A. 80

Persson, B. 347

Pfeiffer, E.F. 29, 386

Pimstone, B. 118, 172

Pister, W. 258

Pogátsa, G. 45

Pointner, H. 303

Portemer, Christiane 372

Prader, A. 381

Puchinger, H. 140, 325

R

Rabkin, R. 172

Radó, J.P. 63, 309

Ragonese, F. 473

Rajaniemi, H. 261

Ranta, J. 58

Raptis, S. 386

Rastogi, G.K. 303

Ratt, L. 67

Remmer, H. 101

Renner, R. 56

Reutter, W. 93

Rogler, J.C. 51

Rohr, R. 222

Roith, D. le 118

Rooyen, R.J. van 231

Rosselin, G. 223

Rossini, A.A. 26

Rupright, M. 65
S

Sailer, S. 444

Salmon, C. 376

Sandhofer, F. 444

Santeusanio, F. 425

Scandellari, C. 230

Scriba, P.C. 258

Scharrer, H. 221

Schatz, H. 29

Schleyer, M. 29

Schmidt, H.J. 180

Schmidt, Th. 386

Schmitz-Moormann, P. 435

Schöffling, K. 9

Schwandt, P. 258

Sehgel, V.K. 160

Serrano-Ríos, M. 57

Singhal, R.L. 139

Sinha, M.K. 303

Smythe, G.A. 227

Solbach, H.G. 474

Söling, H.D. 97

Sonka, J. 286

Spada, A. 289

Sprovieri, Artenisio M. 473

Sraer, J. 232

Stahl, M. 228

Stark, E. 279

Stein, U. 93

Stern, J.S. 37

Stolecke, H. 64

Straková, A. 204, 283

Stubbe, P. 163

Stiuber, G. 386

Sufi, S.B. 62

Suquet, J.-P. 223

Sutter, B.Ch.J. 18, 89

Sutter-Dub, M.-Th. 18

Suzuoki, Z. 333

Szabo, S. 109

T

Taketomi, S. 333

Tiengo, A. 224

Toccafondi, R.S. 62

Travaglini, P. 289

Truffert, J. 461

Tsuda, M. 333

Tuchweber, B. 109

Tung, A.K. 416

Turner, R.C. 241

U

Unger, R.H. 425

V

Vallée, G. 232

Vanha-Perttula, T. 261

Vatier, J. 303

Verdy, M. 59

Vigaš, M. 283, 204, 322

Vincent-Falquet, J.C. 80

Virkkunen, P. 58

W

Wacker, A. 140, 325

Wagner, P. 316

Weir, G.C. 241

Weisweiler, P. 258

Werner, Sigbritt 292

Whitfield, J.F. 200

Wiegelmann, W. 479

Williams, R.H. 250 
Wolf, H. 163, 235

Woodhead, S. 235

Wynn, V. 184, 275
Y

York, D.A. 355

Young, M.K. 213
Z

Zaccaria, M. 230

Zimmermann, H. 474

Zimmermann-Telschow, H. 474 


\section{SUBJECT INDEX}

A

Acetoacetate, insulin secretion 313

Acetyl-CoA 350

Acid base balance 163

Acromegaly, blood amino acids 474

ACTH 258

- biological activity 142

-, bioassay 258,368

- deamidation 142

- effect of collagenase 228

-, in plasma of adrenalectomized rats 368

- neosynthesis and release 306

- secretion and biosynthesis of insulin 29

- transcortin 279

- treatment of rats 279

Adenine 216

Adenine nucleotides, pancreatic islets 141

Adenolypophysis, demonstration of lipotropic hormone by immunofluorescence 229

Adenosine 216

Adenyl cyclase 196

- cerebral 139

-, ovary 376

-, rat heart 330

Adenylate cyclase activity

- inhibition by NSILA-S 56

- myometrium 213

Adipocyte 428

Adipocyte glucose metabolism 37

Adipocytes, fatty rats 355

Adipose cellularity 37

Adipose tissue, actions of HCS 347

_ - , daily meal 440

_ -, deposition of newly synthesized fatty acid 449

- - , glucose uptake 245

-- , rat 245

ADP 216

Adrenal medulla, uptake of tritiated serotonin 466

Adrenalectomy 21

-, ACTH in rat plasma 368

- liver enzymes 204

- lipolysis 292

- rats 283

Adrenaline 204

- urinary excretion during pregnancy 207

Age dependency 93

-, ovarian adenyl cyclase 376

Alanine, glucagon secretion 425

Alloxan, acute effects on the beta cell 404

- calcium metabolism 51

-, diabetes 333

Alpha adrenergic blockade, insulin release 322

- methyl-L-Dopa 26

- MSH 143

Amantadine, growth hormone secretion 454

Amiloride hydrochloride 55

Amino acids

- insulin 54
Amino acids, response to insulin in acromegaly 474

AMP 216

An aerobic glycolysis, beta cells 404

$5 a$-androst-16-ene-3-one 307

Anorexia nervosa 41

Antibodies, insulin 1, 67

- proinsulin-specific 1

Antidiuretic action 309

- effect of clofibrate 461

Antiinsulin serum, action on lipoiysis 432

Antilipolysis, NSILA-S. 56

Arginine, insulin secretion 21

- provocative test 106

Arteriovenous shunting, pulmonary 65

ATP 216

ATPase activity 61

Autoradiography 172

Avian glucagon 416

B

Beta cell, effects of alloxan 404

_ - , insulin release 387

Big and little insulin 70

"Big" insulin 310

Bile, clearance 70

- insulin 70

Binding proteins

- for sexual hormones 271

Bioassay, ACTH 368

Biological action 89

Biosynthesis, glucagon 416

-, insulin, foetal and newborn rats 410

Blood flow, action of HCS 347

Blood glucose

- - in rats 14

- - sex dependence 361

- - sex differences 14

Blood sugar, postprandial 222

C

Caffeine 216

Calcitonin 297

- gastrointestinal stimulation 235

- glucose uptake 145

- in sheep 475

- kinetics of salmon and human 232

- radioimmunoassay 231

- radioiodinated 232

- like activity 297

${ }^{45} \mathrm{Ca} 118$

${ }^{47} \mathrm{Ca} 118$

Calcium metabolism, alloxan 51

- excretion 124

- kinetics 118

- xylose uptake 113

- dependence 292

- dependency of thymic lymphoblast DNS synthesis and proliferation 200

- retention, parathyroid hormone 292

Caloric intake, fractionation 222

Canrenone 312

Carbamazepine 63, 309
Carbohydrate metabolism, liver 45

Carboxypeptidase 93

Catecholamines, insulin release 322

Cathepsin A, D 93

Central nervous system, insulin secretion 21

Cerebral adenyl cyclase and phosphodiesterase 139

\section{cGMP 216}

Chicken 428

Chlorpropamide 45, 309

Cholecystectomy 70

Cholecystokinin-pancreozymin, calcitonin 235

Cholesterol, turnover 444

Citrate 350

Clofibrate, antidiuretic effect 461

Clotrimazol 234

CoA 216

Cold Exposure 286

Collagenase 228

Contraception 134

Contraceptive steroids 184

Corticosteroid treatment, growth hormone secretion 106

Corticosterone, biological half life and distribution volume 279

Corticosterone, daily meal 440

- foetal rat plasma 66

- half time 283

-, insulin release 322

- plasma 204, 283

Cortisol, antagonism to female sex hormones 372

- determinations 63

- effect of testosterone oenanthate on 271

- free 64

- in urine of infants and children 64

- influence on lymphoblasts in thymus 200

-, pyridoxal phosphate-dependent enzymes 372

Cortisone acetate, lipolysis 292

- treatment, rats 34

C-peptide sequences 53

Crop sac stimulation activity 129

Crystallized insulin 395

Cyclic AMP 216

- - phentolamine 391

-, , rat heart 330

- - urine 297

- - phosphodiesterase, rat heart 330

Cyproheptadine, growth hormone secretion 227

Cyproterone acetate 109

D

Deamidation 142

Dehydroepiandrosterone 286

Dehydrogenases, pancreas 435

Denervation 245

2-deoxyglucose, insulin secretion 21

Dexamethasone, suppression of growth hormone release 225

- suppression of the hypothalamic-pituitary-adrenal axis 307

- TSH response 289

Diabetes, cyclic AMP system in rat heart 
Diabetes, early insulin release 387

- free serum insulin 67

- insulin binding in serum 67

- insulin binding to IgG 147

- KK mice 333

- maturity-onset 391

- insipidus, clofibrate 461

Diabetic ketoacidosis, inorganic phosphate of plasma 471

Diabetics, proinsulin-specific antibodies 1

Diabetogenesis, $\mathrm{GH}$ and cortisone 34

Diaphragm muscle 89

Diet, KK mice 333

DIT and calcitonin 475

Diurnal variation, testosterone in plasma 233

DNS synthesis and proliferation 200

Dog 191

-, growth hormone 470

DOPA, growth hormone secretion 454

Dopamine 454

Down's syndrome 57

Drug metabolism 109

E

Electrolyte distribution, insulin content of the pancreas 401

Enzyme induction 109

- protein synthesis 283

Enzymes of energy producing metabolism 435

Epinephrine, effect on adenylate cyclase activity of myometrium 213

- pulmonary arteriovenous shunting 65

Equipotent doses of sulfonylurea drugs 9

Ethionine 435

Excrcise, growth hormone release 225

-, insulin release from periphery 316

Experimental diabetes mellitus 404

Extrapancreatic effects of sulfonylureas 9

Extrapigmentary effect of $a$-MSH 143

F

Fat cells 253, 305

Fatty acids 449

- -, even and odd numbered 191

Fatty rats 355

Fecal excretion 124

Fibroblast mitogen 340

Filter materials for insulin immunoassay 140

Fistula 70

Fluorescence in cortisol determinations 63

Free fatty acids 184

- - - effect of methandienone on 275

--- , glibenclamide 303

_ $\ldots$, insulin secretion 313

- - - uptake during exercise 316
Free insulin 67

Foetal rats, insulin biosynthesis 410

Follicle-stimulating hormone binding proteins 261

Food intake, neonatal mice 223

FSH, ovarian adenyl cyclase 376

- release 266

G

Galactosamine 93

Galactose, insulin in portal vein 4

Gastrectomy 70

Gastrin, fecal and urinary secretion 124

- in pancreas 303

- , levels after "sham eating" 386

-, lipolysis 428

- radioimmunoassay 303

Gastrointestinal hormones, calcitonin 235

Gestation 18

Glibenclamide 9, 303

- blood glucose in rats 14

- maturity-onset diabetics 160

-, sex linkage 361

Glibornuride 9

Glomerular clearance, insulin 75

- filtration, growth hormone ${ }^{125} \mathrm{I}$ 172

Glucagon 196

- adipose tissue 245

- antiserum $30 \mathrm{~K} 241$

-, avian 416

-, biosynthesis 416

-, effect of secretin on 250

- , inhibition of secretion by secretin 425

- radioimmunoassay, interference by plasma 241

-, secretion following alanine stimulation 425

- synthetic (1-23) 60

- antibodies 154

- secretion, pancreozymin 425

Glucocorticoids, TSH 289

Glucodeprivation, cerebral 21

Gluconeogenesis, KK mice 333

Glucoreceptor 325

Glucose 81, 191

-, blood levels in acromegaly 474

-, glibenclamide 303

- in blood of rats 14

-, insulin in portal vein 4

-, insulin release, early phase 387

-, neonatal food intake in mice 223

-, effects on pancreatic islets 410

-, permissive role on the insulinotropic effect of ketone bodies 313

-, pregnant rats 18

-, secretin 250

-, uptake by adipocytes 432

- oxidation, beta cells 404

- 6-phosphate dehydrogenase activity 286

- tolerance, following immunization with insulin 395

- tolerance, GH and cortisone 34

- - test, maturity onset diabetes 391

- uptake, calcitonin 145

_ -, adipose tissue 245
Glucose uptake, exercise 316

- utilization, action of HCS 347

Glycerol 163

- , insulin secretion 313

Glycogen 80

- , liver 204, 283

Glycolysis, liver of KK mice 333

Gonadotropins, ovarian adenyl cyclase 376

Growth hormone, amantadine 454

- - arginine 106

- - effect of collagenase 228

- effect of propanolol on secretion 473

- - , fibroblasts 340

_ - fluctuations 163

- - glibenclamide 303

-,${ }^{125}$ I 172

- _ L-Dopa 41

- , levels in vitamin $B_{6}$-deficient rats 457

- -, mother and child 342

- -, plasma disappearance in the dog 470

- - protein synthesis 167

_- rat adipose tissue 37

- - secretion and biosynthesis of insulin 29

- - secretion in children 106

- - secretion in hypertriglyceridemia 224

- $\quad$ serotonin 227

- suppression with dexamethasone 225

- factors, fibroblasts 340

- inhibition 129

Guanidine group, insulin secretion 55

Guanine 216

Guanosine 216

Gut glucagon-like immunoreactivity 428

H

Hashimoto's thyroiditis 176

HCG 342

HCS, actions in adipose tissue 347

Heart, ATPase activity 61

-, lipoprotein lipase activity 440

Hepatic enzymes, KK mice 333

Hepatitis 93

Hexobarbital 109

Hog pituitaries 258

HPL 342

Human chorionic somatotrophin 29

- placental protein 129

Hypercholesterolemia 444

Hyperglycemia 21

Hyperinsulinism 34

Hyperkalemia 84

Hypersomatotropism, rats 34

Hypertriglyceridaemia 184,275

- with hormone secretion 224

Hypocalcemia, parturient 297

Hypoglycemia 45

Hypogonadism 271

Hypophosphatemia 297

Hypophysectomy 109

- insulin biosynthesis and secretion 29

- liver enzymes 204

- protein synthesis 167

- xylose uptake 113 
Hypothalamic-pituitary, adrenal axis 307

Hypothalamus, LH and FSH release 266

- pituitary, thy roid 289

- ventromedial lesions 48

Hypoxanthine 216

I

Ig-G insulin antibodies 57

Immune reaction, crystallized insulin 395

Immunological tolerance 395

Immunoreactivity, secretin 237

Inanition, rats 457

Influx rate 184

Injury, inhibition of insulin release 322

Inosine 216

Intestinal hormones, lipolysis 428

Insulin 163, 191

-, action, KK mice 333

-, action on lipolysis 432

-, antibodies $1,67,154,395$

-, antibodies, in Down's syndrome 57

- big, little 70

- bile 70

-, binding by IgG 147

-, biosynthesis, foetal and newborn rats 410

- -, GH, ACTH, HCS 29

- caloric intake 222

- cerebral adenyl cyclase and phosphodiesterase 139

-, chromatographed 395

-, content, potassium deficiency 401

- crystalline 75

-, cyclic AMP system in rat heart 330

-, daily meal 440

- effect of secretin on 250

-, immunoassay, filter materials 140

- following glucose, galactose, xylitol and tolbutamide 4

-, fractions 395

- free 67

-, immunoreactive, glibenclamide 303

- interaction with amino acids 54

-, monocomponent 395

- neonatal food intake in mice 223

-, peripheral release 316

- polymerization 75

-, portal vein 4

- potassium transfer 84

- pregnant rats 18

- proteolysis and glucose uptake 89

- release, beta cells, effects of alloxan 404

- - early phase 387

- -, inhibition by injury 322

_, single cell preparation 325

- -, trypsin treatment of islets of Langerhans 325

_ - amiloride hydrochloride 55
Insulin secretion, arginine 21

- - central nervous system 21

- - -2-deoxyglucose 21

- -, effects of alloxan 404

- - GH, ACTH, HCS 29

- - guanidine group 55

- - , ketone bodies 313

- - L-Dopa 26

- - triiodothyronine 230

- $\quad$ Xylitol infusion 221

- serum 70

Insulitis 395

Integral of blood glucose decrease 9

- - insulin increase 9

Interference factors 241

Iodinated hormones, calcitonin 475

Ischemia, pancreatic islets 141

Isolated islets, foetal and newborn rats 410

Isolated islets, glucagon biosynthesis 416

Islets of Langerhans, hypophysectomy 29

- - - L-Dopa 26

_ _ $\quad$ single cell preparation 325

Isolated fat cells 196

_ _ - , lipolysis 432

Isolation of rat islets 325

K

Ketone bodies 191

- -, insulinotropic effect 313

KK mice 333

L

Lactate 191

L-Dopa, growth hormone levels in $\operatorname{man} 41$

- insulin secretion 26

Leucine, carbohydrate metabolism of the liver 45

Leucocyte migration inhibition test 176

Leucocytes 216

LH 134

-, ovarian adenyl cyclase 376

- release 266

- releasing factor 266

- releasing hormone 64

Lipid mobilizing peptide fractions 258

Lipids in serum, caloric intake 222

Lipocyte ghosts 56

Lipogenesis 449

- fatty rats 355

- KK mice 333

Lipolysis 258

- denervation 245

- fatty rats 355

- gastrointestinal hormones 428

- gut glucagon-like immunoreactivity 428

- isolated fat cell 432

- parathyroid hormone 292

- pituitary gland 253

- synthetic glucagon 60

- VIP 196
Lipolytic activity, serum 305

Lipoprotein lipase activity, heart 440

Lipotropic hormone 229

Lipotropin 258

Little and big insulin 70

Liver, glycogen 283

- metabolism 350

- perfusion 101

- protein synthesis 167

- rats 97,101

- tyrosin aminotransferase 204, 283

- ultrastructure 109

Low density lipoprotein 275

L-tyrosine 26

Luteinizing hormone, binding proteins 261

Lymphoblast, DNS synthesis and proliferation in thymus 200

Lysosomal enzymes 93

M

Magnesium, excretion 124

Mammary gland 129

Mannose oxidation, beta cells 404

Maturity-onset diabetes 391

Meal-feeding 440

Median eminence extracts, ACTH 306

Melatonin, growth hormone secretion 227

Mestranol 101

- demethylation 234

Methandienone 275

Methylthiouracil 286

$\mathrm{Mg}^{2+}, \mathrm{ATP}$ ase activity 61

MIT and calcitonin 475

Monoamine-mediated mechanism, diabetes 391

Monocomponent insulin 395

MtT-W15 tumor 34

Muscle, metabolism 316

- protein synthesis 167

Muscular exercise 81

Myometrium 213

$\mathbf{N}$

$\mathrm{Na}^{+}-\mathrm{K}^{+}$-ATPase 312

Neonate food intake, mouse 223

Neutral fat, decrease of "big" insulin after infusion of 310

Newborn infants 163

- rats, insulin biosynthesis 410

Niamide administration, effect on excretion of catecholamines 207

Noble-Collip drum trauma 322

Noradrenaline, grow th hormone secretion 454

-, urinary excretion during pregnancy 207

NSILA-S 56

Nucleotides 216

o

Obese females, plasma renin 59

- hyperglycemic mice 404

Obesity, growth hormone secretion 473

- KK mice 333

Oral glucose tolerance, caloric intake 222

Ovary 216

- adenyl cyclase 376

Oxidation rate 80

Oxytocin 213 
$\mathbf{P}$

Pair feeding, rats 457

Pancreas, effect of ethionine 435

- gastrin 303

Pancreatectomy 84

Pancreatic islets, pyridine nucleotides 141

Pancreozymin, calcitonin 235

- glucagon secretion 425

- lipolysis 428

Paramortal period 163

Parathyroid hormone 51

- - lipolysis and calcium accumulation 292

- - Vit. D deficienty rickets 381

Parkinsonism 41

Pentagastrin, calcitonin 235

- effects in sheep 124

Pentose phosphate cycle, KK mice 333

Peptide mapping 416

Perinatal rats, insulin biosynthesis 410

Peripheral release of insulin 316

Peritoneal dialysis 180

Phenoxybenzamine, insulin release 322

Phentolamine, insulin concentration 391

- insulin release 322

Phosphate, inorganic, in diabetic ketoacidosis 471

Phosphodiesterase, cerebral 139

- rat heart 330

6-Phosphogluconate dehydrogenase activity 286

Phosphorus, excretion 124

Pinealectomy 466

Pituitary gland, binding of thyroid hormone 62

_ _ LH and FSH release 266

- - lipolytic activity 253

Placental protein 129

Polyamine me tabolism 97

Pool sizes, cholesterol 444

Portal vein catheterization 4

Post-heparin lipolytic activity 184 , 275

Post-partum period, urinary excretion of catecholamines 207

Potassium, deficiency, insulin content 401

Potassium, serum and tissue 84

Prediabetes, early phase of insulin release 387

Pregnancy, serum progesteron and insulin levels in rats 18

- urinary excretion of catecholamines 207

Pregnant Rhesus 213

Premature infants 163

Progestagens 134

Progesteron, pregnant rats 18

proinsulin, biosynthesis in foetal and newbom rats 410

- specific antibodies 1

Propanolol, HGH secretion 473

Proteases 89

Protein binding, LH and FSH 261

- synthesis 29

- synthesis, growth hormone 167
Proteinuria 75

Proteohormones 342

Proximal tubular adsorption, growth hormone ${ }^{125}$ I 172

Puerperium 342

Pulmonary arteriovenous shunting 65

Purine derivates 216

Pyridine nucleotides, pancreatic islets 141

Pyridoxal phosphate dependent enzymes 372

Pyruvate 191

\section{$\mathbf{R}$}

Radioimmunoassay, $5 a$-androst-16ene-3-one 307

- calcitonin 231

- gastrin 303

- glucagon 241

- insulin 70

- secretin 237

Radioimmunoelectrophoresis 147

Rat adipose tissue 245

- foetal, corticosterone 66

- heart 61

- liver 45

- a-MSH 143

86 pituitary glands 253

${ }^{86}$ Rb-uptake 312

Reesterification, denervation 245

Refeeding, polyamine metabolism 97

Regitine ${ }^{\circledR}$ insulin concentration 391

Renin in plasma of obese females 59

Reserpine 204

- effect on excretion of catecholamines 207

- insulin release 322

Respiratory distress syndrome 163

Rickets 381

$\mathbf{S}$

Salmon calcitonin 232

Secondary failure 160

Secretin 196

- antibodies to 237

- bioactivity 237

- effect on glucagon 250

- effect on insulin 250

- effect on pancreatic glucoregulatory hormones 250

- inhibition of glucagon secretion 425

- glucose 250

- lipolysis 428

- natural and synthetic 237

- semipurified 237

Serotonin growth hormone secretion 227

- uptake of the adrenal medulla 466

Serum lipolytic activity 305

- induced lipolysis 432

Sex differences, blood glucose 14

- hormones, female, antagonism to cortisol 372

linked blood glucose pattern 361

Sexual hormone binding globulins 271
Sham eating 386

Sheep effects of gastrin 124

Shivering 80

Shunting, arteriovenous due to epinephrine 65

Side effects, glibenclamide 160

Sieving coefficient 75

Sodium uptake, $a$-MSH 143

Somatomedin 340

Somatotropin, xylose uptake 113

Starvation 350

- polyamine metabolism 97

Streptozotocin diabetes 330

Stressed and non-stressed rats 368

Strumigens 286

Sulphonylureas 160

- equipotent doses 9

Sympathetic nervous system 21

Sympatholy tics 41

Synthetic C-peptide sequences 53

$\mathbf{T}$

$\mathrm{T}_{3}$ and calcitonin 476

$\mathrm{T}_{4}$ and calcitonin 475

Temperature 163

Testosterone, diurnal variation in plasma 233

- effect on LH and FSH release 266

- oenanthate 271

Thymidine factor 167

Thymus, lymphoblast DNS synthesis and proliferation 200

Thyroid autoimmunity 176

Thyroid extracts 286

- hormones and calcitonin 475

- - binding to the anterior pituitary gland 62

- secretion rate 48

Thyroglobulin antibodies 176

Thyrotoxicosis 180

Thyrotropin 289

- releasing hormone 289

Thyroxine 271

Thyroxin and calcitonin 475

Thyroxine elimination by peritoneal dialysis 180

Tissue culture, fibroblasts 340

- oxygenation in diabetic ketoacidosis 471

Toad, $a$-MSH 143

Tolbutamide 9

- insulin in portal vein 4

- load, growth hormone secretion 473

Toxicity, glibenclamide 160

Tracer binding radioimmunoassay 231

Transcortin 279

Transumbilical portal vein catheterization 4

Trauma 283

- liver enzymes 204

Triglycerides 184

- short and medium chain 191

- transport 449

- turnover 275

Triiodothyronine insulin secretion 230

Trypsin, islets of Langerhans 325

TSH in mother and child 342

- labelling with ${ }^{125}$ I 58

Tubular reabsorption 75

Turnover, cholesterol 444

- rate 80,184 
Turnover rate, triglycerides 275

Tyrosine amino-transferase, liver 204, 283

U

Ure ter ligation 84

Urinary excretion 124

V

Vasoactive intestinal polypeptide 196

Vanylmandelic acid, urinary excretion during pregnancy 207
Ventromedial hypothalamic lesions 48

Very low density lipoprotein 184, 275

VIP 196

Vitamin $\mathrm{B}_{6}$ deficiency, growth hormone 457

Vitamin D 381

W

Water deprivation test 461

Water excretion 124

Weight, caloric intake 222
$\mathbf{X}$

Xylitol, infusion, insulin secretion 221

- insulin in portal vein 4

Xylose uptake by diaphragm 113

$\mathbf{Y}$

Yellow KK mice 333

$\mathbf{Z}$

Zoxazolamine 109 
Horm. Metab. Res. 5 (1973) 258-261

(C) Georg Thieme Verlag, Stuttgart

\title{
Biological ACTH Activity of Lipid Mobilizing Peptide Fractions from Hog Pituitaries*
}

\author{
O.A. Müller, W. Pister, P. Schwandt, P.C. Scriba and P. Weisweiler \\ I. and II. Medizinische Klinik der Universität München, München, Germany
}

\section{S u m m a r y}

The ACTH activity of lipid mobilizing fractions from porcine pituitaries was determined using a sensitive bioassay for ACTH. For different lipolytic fractions between 12 and 27 ng ACTH per $100 \mu \mathrm{g}$ were found. However, no lipolytic response was obtained in the rabbit with approximately 2,5 $\mu \mathrm{g}$ АCTH. It was therefore concluded, that ACTH does not contribute significantly to the lipolytic activity of our lipotropin preparations. The preparations appear however to contain a minimal ACTH contamination, which was lost upon storage of solutions at $-16^{\circ} \mathrm{C}$ with preservation of the lipolytic activity.

*Supported by the Deutsche Forschungsgemeinschaft (SFB 51)

Received: 20 June 1972

Accepted: 12 Mar. 1973
Key-Words: Hog Pituitaries - Lipotropin - ACTH - Lipolysis - ACTH-Bioassay

\section{Introduction}

$\mathrm{ACTH}$ was shown to have considerable intrinsic lipid mobilizing activity in several species tested (Lebovitz and Engel 1965). Hence, it is necessary to determine the ACTH activity in all specific lipid mobilizing peptides purified from pituitaries (lipotropins).

The ACTH activity of a lipotropin fraction, peptide A, which was further purified in our laboratories (Schwandt, Karl, Thüner and Knedel 1968, Schwandt, Weisweiler and Lamerz 1971), was studied by a sensitive bioassay. The results are given in this paper. 


\section{Materials and Methods}

\section{Purification of lipid mobilizing peptide fractions}

Fraction $\mathrm{G}$ was extracted from lyophilized hog pituitaries, as published (Schwandt et al. 1968). Further purification resulted in fraction J and peptide A (Schwandt et al. 1971). The three top fractions of the Sephadex-G-50 rechromatography of peptide $A$ were here defined as A peak.

\section{Biological ACTH activity}

The increment of corticosterone output into the adrenal venous blood of acutely hypophysectomized rats was used for bioassay of ACTH activity (Scriba, Hacker, Dieterle, Kluge, Hochheuser and Schwarz 1966, Pister 1972). Standards $\left(\beta^{1-23}\right.$-corticotropin amide, Acethropan ${ }^{\circledR}$ S, Farbwerke Hoechst) and test solutions were given intraaortally. The sensitivity and reproducibility of the method were improved, chiefly by introduction of a spectrofluorimetric analysis for corticosterone (Pister 1972), which is similar to our cortisol method (Scriba, Gerb, Kluge, Boss and Müller 1970): Less than $0.005 \mu \mathrm{g}$ corticosterone per $0.1 \mathrm{ml}$ could be determined. The standard curve for ACTH (Fig. 1) shows a linear relation between dosis ACTH $(1 \mathrm{ng}=100 \mu \mathrm{U})$ and corticosterone increment. This linearity was found up to doses of $22 \mathrm{ng}$ ACTH. On the other hand $0.05 \mathrm{ng} \mathrm{ACTH}$ $(=5 \mu \mathrm{U})$ gave a significant rise of corticosterone output (Pister 1972).

\section{Lipolytic activity}

The lipolytic activity of the various lipotropin fractions and of Acethropan ${ }^{\circledR}-S$ was tested in male and female rabbits, $2.5 \mathrm{~kg}$ of weight (Grauscheck, single cages, Altromin ${ }^{\circledR}$-pellets ad libitum). Blood was taken for the determination of FFA (Dole and Meinertz 1960) from an ear vein before and 1 hour after subcutaneous injection of $250 \mu \mathrm{g}$ of fractions $\mathrm{G}$ and $\mathrm{J}$, of peptide A and of $2.5 \mu \mathrm{g} \mathrm{ACTH}$. All test substances dissolved in $0.9 \% \mathrm{NaCl}$ solution.

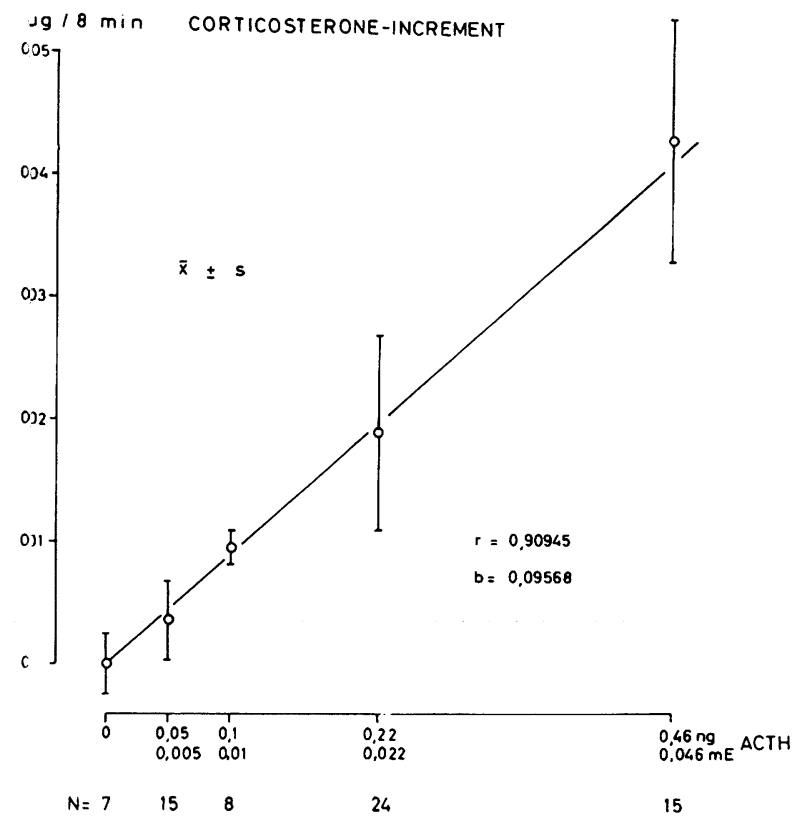

Fig. 1. ACTH standard curve.

$\mathrm{N}=$ number of rats, $\mathrm{r}=$ correlation, $\mathrm{b}=$ regression.

For further details see text.

\section{Results}

The ACTH activities determined in the different lipid mobilizing fractions varied between 12 and $27 \mathrm{ng}$ ACTH per $100 \mu \mathrm{g}$ of the lyophilized fractions as for peptide $A_{\text {peak }}$ and for fraction $J$ respectively (Table 1). Thus, on a weight basis a ratio of 1:10 000 was calculated for peptide $A_{\text {peak. Th }}$. This result was confirmed for all fractions by determination of the ACTH activity in $10 \mu \mathrm{g}$ amounts. Further the fraction with the lowest ACTH activity (peptide $A_{\text {peak }}$ ) was tested for 5 and $1 \mu \mathrm{g}$ amounts with the result of a dilution curve, the slope of which was very similar to the ACTH standard curve (Table 2). These data had been obtained with lyophilized fractions that had been stored for 2 to 3 weeks at $-16^{\circ} \mathrm{C}$. No loss of activity was seen when peptide $\mathrm{A}$ was stored for 4 months at $-16^{\circ} \mathrm{C}: 18.56 \pm 1.47 \mathrm{ng}$ ACTH per 100 $\mu \mathrm{g}$ vs. $16.45 \pm 1.31$ in fresh material (Table 1 ); However, storage of solutions of the lipid mobilizing fractions for 9 weeks at $-16^{\circ} \mathrm{C}$ resulted in complete loss of the ACTH activity, whereas the lipolytic activity was not diminished.

\section{Discussion}

Undoubtedly ACTH activity was demonstrated in all the lipid mobilizing fractions tested: however, ACTH accounts only for 0.1 to $0.3^{\circ} \% 0$ of the amount assayed. Acethropan ${ }^{\circledR}-S\left(\beta^{1-23}\right.$-corticotropin amide) given in 100 -fold excess of this did not elevate the FFA (Table 1). The same was shown (Schwandt et al. 1968) for equivalent amounts of porcine ACTH $(0.4 \mathrm{U})$. Therefore, the minimal ACTH activity in our lipid mobilizing fractions cannot explain their lipolytic activity.

On the other hand, one cannot definitively answer at present the question, whether the small ACTH activity of the fractions is due to ACTH contamination or intrinsic ACTH activity of the lipotropins. The specificity of the ACTH bioassay (Schleyer, Evertz, Voigt, Fehm, Faulhaber and Pfeiffer 1970) and the similar slope of the ACTH standard curve and of the lipotropin dilution curve are both in favor of the ACTH contamination theory, since a non-parallel slope of the dilution curve should have been expected for the case of intrinsic ACTH activity in lipotropins or in fragments of lipotropins. This view is further supported by the observation, that with purification of the lipid mobilizing fractions the ACTH activity per $\mu$ g injected substance decreases with increasing lipolytic activity. Finally, the observed loss of ACTH activity with preservation of the lipolytic activity in solutions kept at $-16^{\circ} \mathrm{C}$ is clearly in favor of the assumption that we are dealing with ACTH contaminations. The disappearance of the biological ACTH-activity in solution at $.16^{\circ} \mathrm{C}$ was demonstrated in own experiments (Pister 1972) and has also been shown in pilot experiments 
Table 1. Protein content as $\%$ weight $(\mathrm{N}=$ number of tested preparations), lipolytic and ACTH activity $(\mathrm{N}=$ number of the animals tested) of the lipid mobilizing fractions.

$\triangle F F A=$ increase of the free fatty acids.

\begin{tabular}{|c|c|c|c|c|}
\hline Fraction & $\begin{array}{c}\% \text { Protein } \\
\text { (Lowry-method) } \\
\text { mean } \pm \mathrm{SD}\end{array}$ & $\begin{array}{c}\Delta \mathrm{FFA} \\
\text { mval/1 } \\
\text { mean } \pm \mathrm{SD}\end{array}$ & per $100 \mu \mathrm{g}$ & $\begin{array}{l}\mathrm{g}=1 \mathrm{mE}) \\
\text { per } 10 \mu \mathrm{g} \\
\text { ance } \\
D\end{array}$ \\
\hline G & $\begin{array}{c}58.7 \pm 2.2 \\
\quad(\mathrm{~N}=6)\end{array}$ & $\begin{array}{c}1.51 \pm 0.24 \\
(\mathrm{~N}=11)\end{array}$ & $\begin{array}{c}13.98 \pm 0.51 \\
(\mathrm{~N}=3)\end{array}$ & $\begin{array}{c}1.34 \pm 0.08 \\
(\mathrm{~N}=5)\end{array}$ \\
\hline $\mathbf{J}$ & $\begin{array}{c}77.7 \pm 2.2 \\
(\mathrm{~N}=6)\end{array}$ & $\begin{array}{c}2.99 \pm 0.20 \\
(\mathrm{~N}=6)\end{array}$ & $\begin{array}{c}27.21 \pm 7.31 \\
(\mathrm{~N}=6)\end{array}$ & $\begin{array}{c}1.85 \pm 0.13 \\
(\mathrm{~N}=2)\end{array}$ \\
\hline $\mathbf{A}$ & $\begin{array}{c}99.4 \pm 2.0 \\
(\mathrm{~N}=6)\end{array}$ & $\begin{array}{c}3.28 \pm 0.09 \\
(N=6)\end{array}$ & $\begin{array}{c}16.45 \pm 1.31 \\
(\mathrm{~N}=3)\end{array}$ & $\begin{array}{c}1.71 \pm 0.15 \\
(\mathrm{~N}=3)\end{array}$ \\
\hline$A_{\text {peak }}$ & - & - & $\begin{array}{c}12.35 \pm 2.79 \\
(\mathrm{~N}=3)\end{array}$ & $\begin{array}{c}1.10 \pm 0.13 \\
(\mathrm{~N}=3)\end{array}$ \\
\hline $\begin{array}{c}\text { ACTH } \\
\text { (Acethropan }^{\circledR}{ }_{S}\end{array}$ & - & $\begin{array}{c}0.05 \pm 0.15 \\
(\text { from }-0.11 \\
\text { to }+0.26) \\
(N=5)\end{array}$ & - & - \\
\hline
\end{tabular}

Table 2. ACTH activity in different dilutions of the lipolytic peptide $A_{\text {peak }}$

The decrease of the ACTH activity parallels the diminition of the amount of the added lipolytic material.

\begin{tabular}{ccc}
\hline $\begin{array}{c}\text { lipolytic peptide Apeak } \\
\mu \mathrm{g}\end{array}$ & \multicolumn{2}{c}{$\begin{array}{c}\text { ACTH activity } \\
\mathrm{ng}(10 \mathrm{ng}=1 \mathrm{mE})\end{array}$} \\
& $\begin{array}{c}\text { single values } \\
\text { determined }\end{array}$ & mean $\pm \mathrm{SD}$ \\
\hline 100 & 15.46 & $12.35 \pm 2.79$ \\
& 12.89 & \\
& 8.70 & \\
10 & 1.23 & $1.10 \pm 0.13$ \\
& 1.14 & \\
& 0.92 & \\
5 & 0.72 & $0.71 \pm 0.01$ \\
& 0.71 & \\
& 0.69 & \\
& 0.21 & $0.16 \pm 0.04$ \\
& 0.12 & \\
\hline
\end{tabular}

$\mathrm{R}$ e f e r e n c e s

Cseh, G., L. Gráf, E. Góth: Lipotropic hormone obtained from human pituitary gland. FEBS Letters 2: $42-44$ (1968)

Dole, V.P., H. Meinertz: Microdetermination of long-chain fatty acids in plasma and tissue. J.biol.Chem. 235: 25952597 (1960)

Gráf, L., G. Cseh: Isolation of porcine $\beta$-lipotropic hormone. Acta biochem.biophys.sci.hung. 3: 175-177 (1968)

Lebovitz, H.E., F.L. Engel: In vivo and in vitro adipokinetic effects of corticotropin and related peptides. In: Handbook of Physiology, Sect. 5, Ed. A.E. Renold, G.F. Cashill, jr.: Williams \& Wilkins, Baltimore (Maryland) 1965 , p. $541-547$

Lohmar, P., C.H. Li: Biological properties of ovine $\beta$-lipotropic hormone. Endocrinology 82: 898-904 (1968) by former investigators (Scriba et al. 1966). The basis of this observation remains unclear.

The ACTH activity varies in the different preparations of lipotropins reported. ACTH accounted for 0.2 to $0.3^{\circ} / 00$ of the preparations from porcine and human pituitaries of Rudman, Seidman and Reid (1960), of Graf and Cseh (1968) and of Cseh, Graf and Goth (1968). Schleyer et al. (1970) reported $0.1^{0} \% \mathrm{ACTH}$ in a fraction of weak lipolytic activity (P-LF I) and $1^{0 / 00}$ ACTH in fractions P-LF II and P-LF III of higher lipolytic activity. Similar ACTH activities have been found in lipolytic preparations by Ryschka and Chochlow (1965). Trygstad (1968) reported $2^{0 / 00}$ ACTH for LMF VI from human pituitaries and Lohmar and $\mathrm{Li}$ (1968) $10^{\circ} / 00$ for $\beta$-LPH from ovine pituitaries.

All these authors agree, however, that the lipolytic activity of their lipid mobilizing fraction was not caused by ACTH contamination only. However, a definite discrimination of the lipotropins against all other lipolytic pituitary hormones has not been achieved so far.

Pister, W.: Dissertation, München (1973)

Rudman, D., F. Seidman, M.B. Reid: Lipemia-producing activity of pituitary gland: Separation of lipemia-produiing component from other pituitary hormones. Proc. Soc.exp.Biol. 103: 315-320 (1960)

Ryschka, F.J., A.S. Chochlow: Reinigung und Untersuchung des Lipotropins aus Hypophysen verschiedener Spezies (russisch). Biochimija 30: 1277-1284 (1965)

Schleyer, M., W. Evertz, K.H. Voigt, H.L. Fehm, J.D. Faul. haber, E.F. Pfeiffer: Studies on the pituitary „Fettstoffwechselhormon". III. The corticotropic activity of some lipolytic effective hog pituitary gland fractions in vivo. Horm.Metab.Res. 2: 333-337 (1970)

Schwandt, P., H.J. Karl, J. Thüner, M. Knedel: Untersuchıngen über eine lipolytisch wirksame Substanz aus Schwenehypophysen (Fraktion H). - Extraktion und Reini- 
gung der lipolytischen Substanz. Z.ges.exp.Med. 147: 246-252 (1968)

Sichwandt, P., P. Weisweiler, R. Lamerz: Darstellung eines lipolytisch wirksamen Polypeptids (Peptid A) aus Hypophysen vom Schwein. Z.ges.exp.Med. 155: 195-203 (1971)

Scriba, P.C., R. Hacker, P. Dieterle, F. Kluge, W. Hochhauser, $K$. Schwarz: ACTH-Bestimmungen in Plasma aus dem Bulbus cranialis venae jugularis. Klin.Wschr. 44: 1393-1398 (1966)
Scriba, P.C., A.C. Gerb, F. Kluge, N. Boss, O.A. Müller: Eine verbesserte fluorimetrische Serumcortisolbestimmung. Z.Anal.Chem. 252: 284-287 (1970)

Trygstad, $O$.: The lipid-mobilizing effect of some pituitary gland preparations. II. Preparation of a human pituitary lipid-mobilizing factor (LMF) with hypocalcaemic and hyperglycaemic effects in rabbits. Acta endocr. (Kbh.) 57: 81-108 (1968)

Requests for reprints should be addressed to: Dr. O.A. Müller, II. Medizinische Universitätsklinik, D-8000 München, Ziemssenstr. 1 (Germany) 